Programme (PPP)'. Childbearing potential is defined as a premenopausal female capable of becoming pregnant.

Hospital specialists are required to review females on valproate to establish childbearing potential and only continue if other treatments are ineffective and pregnancy is excluded by means of a serum pregnancy test. PPP should be started if valproate is to be continued ensuring there is understanding of the risks to an unborn child and an Annual Risk Acknowledgement form (ARAF) is completed.

Aim Identify all female paediatric patients taking valproate under the care of a paediatrician in a district general hospital in November 2018 to assess compliance with PPP.

Method 163 female paediatric patients identified via clinic lists of 5 consultants seeing Epilepsy and neurology patients.

Results $12(7.4 \%)$ were taking Valproate (mean age 10; range 4 to 16$) .5$ out of the $12(41.7 \%)$ had a signed ARAF. 1 GP and 1 pharmacist had alerted hospital specialists of 2 of the $12(16.7 \%)$ patients. There was no documentation regarding contraception. 2 patients without an ARAF (16.7\%) were in the process of being weaned off valproate following the MHRA guidance.

Conclusion Following departmental presentation in February 2019, 2 further female patients were weaned off valproate and consultants have been made aware of PPP and familiarised with the ARAF. Concerns were raised by specialists regarding the ethics of contraception in young females that may lack capacity to consent. On the 29th March 2019 A Guidance Document on Valproate Use in Women and Girls of Childbearing Years was released. It provides practical information, guidance and sources of further support to clinicians managing patients on Valproate. Valproate is now under strict regulation and use is expected to be in line with the modified ARAF and updated guidance.

\section{G75(P) PREOPERATIVE BOTULINUM TOXIN A FOR CHILDREN WITH BILATERAL CEREBRAL PALSY UNDERGOING MAJOR HIP SURGERY: A RANDOMISED DOUBLE-BLIND PLACEBO-CONTROLLED TRIAL WITH 6 MONTHS FOLLOW-UP}

${ }^{1} \mathrm{~A}$ Sobti, ${ }^{1,2} \mathrm{~N}$ Magill, ${ }^{2,3} \mathrm{~F}$ Norman-Taylor. ${ }^{1}$ Medical Statistics, London School of Hygiene and Tropical Medicine, London, UK; ${ }^{2}$ Biostatistics, King's Clinical Trial Unit, London, UK; ${ }^{3}$ Paediatric Orthopaedics, Evelina London Children's Hospital, London, UK

\subsection{6/archdischild-2020-rcpch.56}

Aims Cerebral Palsy (CP) describes a group of permanent disorders of movement and posture. Children with CP often have severely displaced and dislocated hips and require hip surgery. These children are in pain post-operatively and are likely to find it difficult to communicate pain due to cognitive and communication impairments. The use of Botulinum Toxin Type A (BoNT-A) injections has been proposed to help reduce the pain and in doing so, improve quality of life (QOL). The objective of the abstract is to model the typical course of pain and QOL over 6 months of follow-up, taking the missing data into consideration.

Methods The trial was a randomised, double-blind, longitudinal trial. Children from a tertiary hospital awaiting hip surgery, allocated to receive either BoNT-A injections or placebo treatment. After surgery, the primary outcome of pain was measured using the paediatric pain profile (PPP) at 9 time points, up to 6 months post-randomisation. The secondary outcome of QOL was measured using the Caregiver Priorities and Child Health Index of Life with Disabilities (CPCHILD) questionnaire at 3 time points, up to 6 months post-randomisation. The pattern and nature of missing data was explored, and a logistic regression method was used to find any characteristics measured at baseline that were predictive of missing data. The data was analysed at each time point using an analysis of covariance (ANCOVA) method and multiple imputation was used to account for the missing data.

Results 54 participants were equally allocated to either the BoNT-A or placebo group. There was a reduction in pain at each consecutive time point in both groups. There was weak evidence to suggest BoNT-A was beneficial compared to placebo treatment at 6 months post-randomisation with an estimated difference of $3.115(p=0.065)$ in PPP Score. However, no statistically significant difference was found at the other time points. All models showed an improvement in QOL over time, but no statistically significant differences were found between BoNT-A and placebo.

Conclusions The trial did not demonstrate a benefit in using BoNT-A to reduce pain or improve QOL postoperatively.

\section{G76(P) MANAGEMENT OF HYPERTONIA IN CHILDREN AND YOUNG PEOPLE (CAYP) WITH INTRATHECAL BACLOFEN (ITB) IN A TERTIARY CENTRE}

${ }^{1} \mathrm{D}$ Chandrasegaran, ${ }^{2} \mathrm{~S}$ Perides, ${ }^{2} \mathrm{~S}$ Barkey, ${ }^{2} \mathrm{DE}$ Lumsden, ${ }^{3} \mathrm{~S}$ Bassi, ${ }^{2} \mathrm{JP}$ Lin, ${ }^{2} \mathrm{M}$ Kaminska. ${ }^{1}$ Acute Medicine, Basildon University Hospital, Basildon, UK; ${ }^{2}$ Complex Motor Disorder Service, Evelina London Children's Hospital, London, UK; ${ }^{3}$ Paediatric Neurosurgery, King's College Hospital NHS Foundation Trust, London, UK

\subsection{6/archdischild-2020-rcpch.57}

Aims To review the indications for and outcomes following ITB for CAYP managed at our centre.

Methods 42 Patients undergoing ITB pump insertion from 2006 to 2015 were identified at our centre. 35 had reports available and were included in a retrospective note review, using a standardised data collection proforma.

Results Median age at ITB pump insertion was 9 (range 4 to 18). Hypertonia was described as dystonia, spasticity and/or dyskinesia. ${ }^{1}$

Median length of follow up was 3 years. Pre-surgery goals determined the choice of outcome measures.

Care Provider Child Health Index Living with Disability (CPCHILD) data, available for 23 CAYP at baseline and 18 CAYP at 1 year, improved from a median score of 45.5 to $58.2(\mathrm{P}=0.03$, Wilcoxon signed rank test).

Burke-Fahn-Marsden Dystonia Rating Scale (BFMDRS) data was available for 12 CAYP with dystonia at baseline (median

\section{Abstract G76(P) Table 1}

\begin{tabular}{ll}
\hline Type of hypertonia & Number of children \\
\hline Spasticity only & 6 \\
Dystonia only & 12 \\
Dyskinesia only & 0 \\
Spasticity + Dystonia & 6 \\
Spasticity + Dyskinesia & 0 \\
Dystonia + Dyskinesia & 4 \\
Spasticity + Dystonia + Dyskinesia & 1 \\
& \\
Missing data & 6 \\
\hline
\end{tabular}


92.5 for motor, 30 for disability) and 10 CAYP at 1 year (median 98.25 for motor, median 28 for disability). Gross Motor Function Measure (GMFM) score was available for 9 CAYP at baseline (median 40), 8 CAYP at 1 year (median 47) and 7 CAYP beyond 1 year (median 43.1). Changes in GMFM and BFMDRS did not reach statistical significance.

Pain was measured with paediatric pain profile, classified into most troublesome pain (MT) and pain on a good day (GD), available for 14 CAYP at baseline and 6 CAYP at 1 year. There was marked improvement in median MT pain (29 at baseline to 17 at 1 year) but less significant improvement in median GD pain (16 at baseline to 13.5 at 1 year).

Conclusions For a heterogenous cohort of CAYP with motor disorders, ITB appeared to improve ease of care and comfort as indicated by change in CPCHILD score. Multiple measures are required to fully capture benefits seen in this cohort, which should be focused on their individual needs for intervention.

\section{G77(P) RESPIRATORY TRACT INFECTION IN CHILDREN WITH A NEURODISABILITY - MICROBIOLOGY, ANTIBIOTIC CONSUMPTION AND HOSPITAL ADMISSION - A SERVICE EVALUATION FOR A HIGH-RISK GROUP}

${ }^{1,2} \mathrm{~L}_{\text {Marrable, }}{ }^{1,2} \mathrm{JRF}$ Sanner, ${ }^{2} \mathrm{M}$ Flanigan, ${ }^{2} \mathrm{~T}$ Wolff, ${ }^{1,2} \mathrm{MN}$ Hurley. ${ }^{1}$ School of Medicine: Division of Child Health, Obstetrics and Gynaecology, University of Nottingham, Nottingham, UK; ${ }^{2}$ Nottingham Children's Hospital, Nottingham University Hospitals NHS Trust, Nottingham, UK

\subsection{6/archdischild-2020-rcpch.58}

Aims Children with neurodisability (ND) have an increased risk of respiratory tract infections (RTI). We aimed to establish the organisms implicated in pulmonary infection in children with ND in our area in the context of antibiotic provision, as well as document hospital admissions within this population.

Methods The City Rapid Access Physiotherapy Service (RAPS) cares for children with ND who have frequent RTIs. A random number generator was used to select a sample of 30 children from a complete list of 74 children accessing this service. Respiratory tract samples are taken when RTI is suspected. Patient records were used to investigate respiratory microbiology, hospital admissions and inpatient antibiotic provision, from $30 / 09 / 15$ to $30 / 09 / 19$ inclusive.

Results Of the 30 children, 15 were male; 14 had cerebral palsy, 6 had a neurodegenerative disease, 5 had a neuromuscular condition and 5 had other complex ND conditions. A total of 184 admissions were documented across 26 patients; the average admission length was 9.5 days (range 0-116 days). The average number of positive respiratory microbiology samples per patient was 8 (range 0-54). 7 patients had no positive respiratory samples. $185(76.8 \%)$ positive isolates were viral and $56(23.3 \%)$ were bacterial. Common isolates are presented in table 1.

18 patients received prophylactic antibiotics, notably azithromycin (16). In total, 176 courses of inpatient antibiotics were prescribed; 36 were co-amoxiclav. The average number of inpatient antibiotics prescribed per child was 6 (range 041). Lower RTI was the most common (46.1\%) documented reason for antibiotic prescription.

Conclusion Viruses are most commonly isolated from children with ND during an exacerbation of symptoms, although many bacteria are also implicated. Infections in these patients lead to frequent inpatient admissions and are managed by a wide
Abstract G77(P) Table 1 Organisms most commonly isolated in respiratory samples from a random sample of 30 children with ND

\begin{tabular}{lll}
\hline Organism & Number of patients isolated in & Number of isolations \\
\hline Rhino/enterovirus & 19 & 113 \\
Parainfluenza virus & 15 & 38 \\
Respiratory syncytial virus & 14 & 37 \\
Human coronavirus & 10 & 18 \\
Adenovirus & 8 & 14 \\
Metapneumovirus & 9 & 12 \\
Pseudomonas aeruginosa & 6 & 17 \\
Haemophilus influenzae & 7 & 15 \\
\hline
\end{tabular}

variety of antibiotics, of which co-amoxiclav is the most commonly prescribed. It is uncertain whether there is an association between the microbiology and severity of disease.

\section{G78(P) ABSTRACT WITHDRAWN \\ G79(P) CYTOMEGALOVIRUS AND HERPES SIMPLEX VIRUS 1 \& 2 MENINGITIS AMONG CHILDREN WITH SUSPECTED MENINGITIS AT A TERTIARY EMERGENCY UNIT IN NIGERIA}

${ }^{1} \mathrm{~A}$ Akindolire, ${ }^{2} \mathrm{~A}$ Adigun, ${ }^{1} \mathrm{O}$ Tongo, ${ }^{1} \mathrm{AO}$ Asinobi, ${ }^{3} \mathrm{~A}$ Odaibo, ${ }^{3} \mathrm{D}$ Olaleye. ${ }^{1}$ Department of Paediatrics, University of Ibadan/University College Hospital Ibadan, Nigeria; ${ }^{2}$ University College Hospital Ibadan, Nigeria; ${ }^{3}$ Department of Virology, University of Ibadan, Ibadan, Nigeria

\subsection{6/archdischild-2020-rcpch.59}

Aim To determine the incidence of cytomegalovirus and herpes simplex virus $1 \& 2$ among paediatric emergencies presenting with suspected meningitis at the children's emergency room.

Methods It was a prospective cross sectional study of all consecutive paediatric emergencies over a period of three months. Patients were included in the study if they had any neurological signs such as but not limited to lethargy, coma seizures with or without fever. Clinical details were entered into a structured proforma. Cerebrospinal fluid (CSF) samples were obtained at admission and sent to the Virology Laboratory in a cold box. Deoxyribonucleic acid (DNA) extraction was done using commercially available DNA extraction kit by Jena Bioscience (Jena Germany) according to the manufacturer's instruction. The DNA was then tested for HSV $1 \& 2$ and CMV using gene specific primers in a multiplex reaction. Positive samples were detected after agarose gel electrophoresis using a trans illuminator.

Results Forty patients were recruited, 29 (72.5\%) males and $11(27.5 \%)$ females, with a mean age of $27.1 \pm 18.3$ months. The most common presenting clinical features were seizures 37 (92.5\%), fever 35 (87.5\%), lethargy 29 (72.5\%), poor feeding $23(57.5 \%)$ and coma $11(27.5 \%)$. Twenty (50\%) the patients had CSF positive for HSV 1, 13 (32.5\%) of whom were also positive for CMV and 12 (30\%) for HSV 2. Eleven patients $(27.5 \%)$ were positive for all three viruses.

Conclusion The high incidence of viral meningitis among children with suspected meningitis is consistent with findings from high income countries. Mortality and morbidity from meningitis remain high in this setting however, viral aetiology 